Annals of International Medical and Dental Research

E-ISSN: 2395-2822 | P-ISSN: 2395-2814

Vol-8, Issue-2 | March-April 2022

DOI: 10.53339/aimdr.2022.8.2.15

Page no- 109-112 | Section- Research Article (Pain Medicine)

\title{
Acupuncture Treatment in Rheumatoid Arthritis Patients in a Specialized Acupuncture Center in Dhaka Bangladesh: A Case Report
}

\author{
S.M. Shahidul Islam ${ }^{*}$, Huihui Li $^{2}$, S.M. Abdul Alim ${ }^{3}$, Sabina Yasmin ${ }^{4}$
}

$1 \mathrm{PhD}$ fellow in pain management, specially trained in Acupuncture, Suo Xi Hospital Limited, Shaan Tower, Chamelibagh, Santinagar, Dhaka, Bangladesh.

Email ID: drshahidul@yahoo.com

Orcid ID:0000-0002-6294-1688,

${ }^{2}$ Consultant, Department of Orthopedics and Traumatology, People's Hospital of Chinese Medicine, Ruian City, China, Email ID: drshahidul@yahoo.com, Orcid ID:0000-0002-6294-1688

3Pain Medicine \& ICU Specialist, Head of Department, Central Police Hospital, Ex. Associate Professor, Ad-din Medical College, Dhaka, Bangladesh, Ex. Medical Incharge UN Mission, Sudan.

Email ID: drsmaalim@gmail.com

Orcid ID:0000-0002-6294-1688

${ }^{4} \mathrm{CEO}$, Suo Xi Hospital Limited, Shaan Tower, Chamelibagh, Santinagar, Dhaka, Bangladesh.

Email ID: drshahidul@yahoo.com

Orcid ID:0000-0002-6294-1688

${ }^{*}$ Corresponding author

Received: 10 December 2021

Revised: 16 January 2022

Accepted: 25 January 2022

Published: 18 February 2022

\begin{abstract}
Background: Two recognized antibodies, Rheumatoid Factor (RF) and Anti-Citrullinated Peptide Antibody (ACPA), are produced in rheumatoid arthritis to target common autoantigens that are expressed in and around the joints. Tobacco and microorganisms, as well as the relationship between genetics and environment, play a critical role in the progression of the illness (e.g. Porphyromonas gingivalis). Rheumatoid arthritis is visualized as a Th1 and Th17 illness in the first stages of the disease. There is a significant role for inflammatory cytokines in the hierarchy of RA processes. Additionally, RA-related joint degradation is mediated by the Wnt system and osteoprotegerin's impact on osteoclasts, as well as the matrix synthesis dysregulation that causes cartilage degeneration. Rheumatoid arthritis is a disease in which both innate and adaptive immunity have been shown to play a key role, thanks to the development of effective therapies for TNF, IL-6 receptor, IL-1, CD20 B cells, and T-cell/Dendritic cell interactions.
\end{abstract}

Keywords:- Rheumatoid, Arthritis, Diabetic, Physiotherapy

\section{INTRODUCTION}

Inflammatory arthritis, often known as rheumatoid arthritis (RA), is characterized by synovitis that has been consistently and erosive degraded, as well as extra-articular involvement in certain cases.[1] Having a chronic fluctuating disease is frequent in persons, ${ }^{[2]}$ and it may cause joint deterioration and deformity as well as disability and even death if left untreated. In the United States, RA is responsible for an estimated 9 million medical visits and 250,000 hospitalizations each year.[3,4] Disabilities caused by RA may have a significant impact on a family's financial wellbeing and quality of life. Adults in the United States are diagnosed with RA at a rate of less than one percent. Because the condition is so uncommon, most physicians only receive a limited amount of exposure to it throughout the course of their professional lives. Based on the presumption that an accurate diagnosis of RA has been established, the following 
recommendations for treatment of RA are made. $[5,6]$

\section{CASE REPORT}

Our hospital was visited by a 55-year-old female patient from Joypurhat, Bangladesh, who had complained of numerous joint aches. She was admitted to our hospital (both large \& small joints). He had been in excruciating agony for the better part of the day. We begin by doing a routine blood test on the patient to determine his or her condition. The patient had been diabetic for the previous three years, and the condition was managed with medication. We begin to look into her family history, but we discover that there is nothing remarkable in her family background. Acupuncture, Chinese therapy (deep muscle stimulator and multifunctional equipment) and Physiotherapy were included in our treatment plan for the patient's leg and shoulder (Mobilization, manipulation, TENS, IRR).multi-functional equipment) and Physiotherapy were included in our treatment plan for the patient's leg and shoulder (Mobilization, manipulation, TENS, IRR).

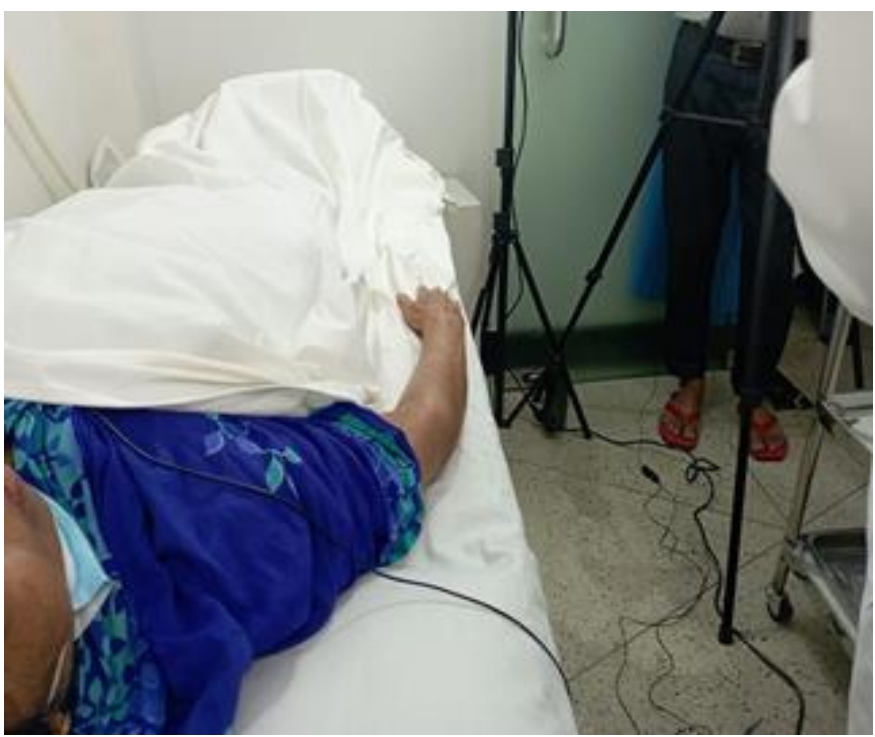

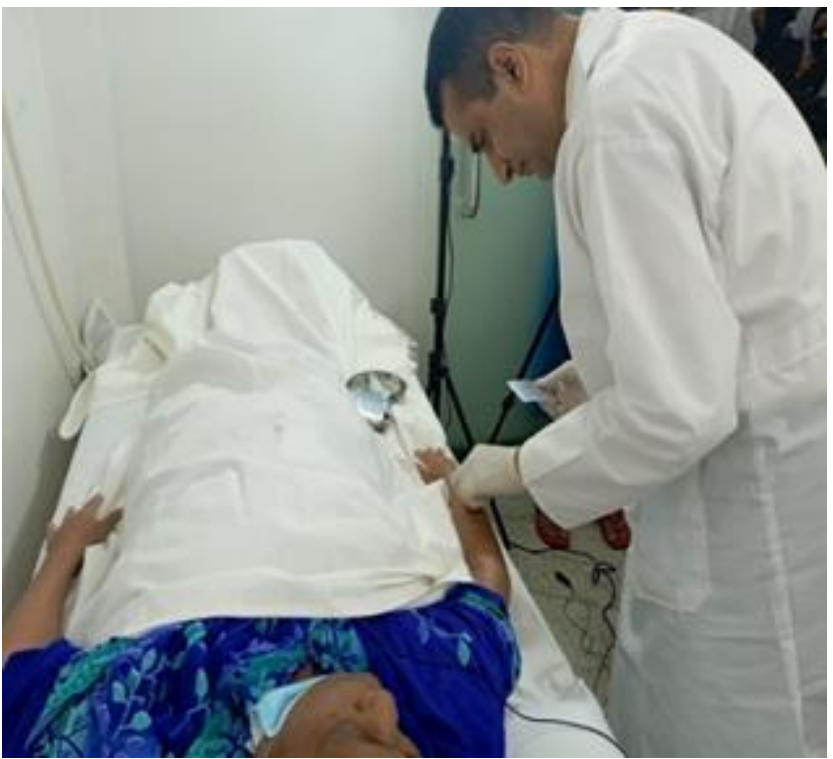

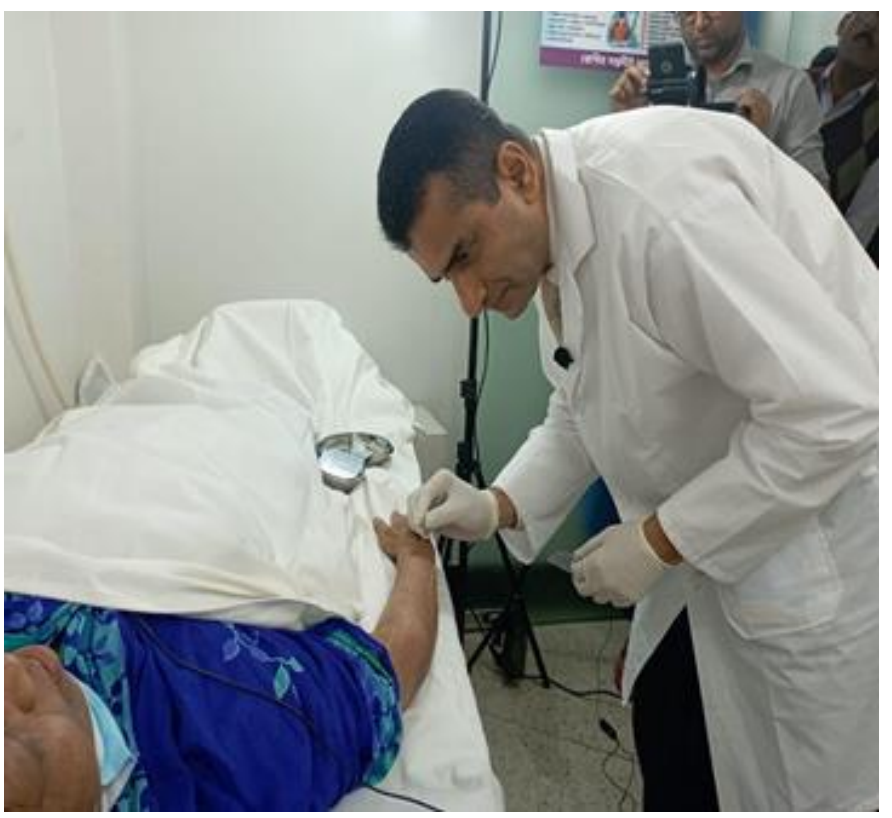

Figure 1 a,b,c: Giving Acupuncture on both leg and shoulder

Only a few numbers of natural treatments are as powerful as tongue acupuncture (TA). Acupuncture points on the tongue are said to connect to certain parts of the human body. For example, it is said that TA may influence how the body's blood and energy flow by activating 
Annals of International Medical and Dental Research E-ISSN: 2395-2822 | P-ISSN: 2395-2814

Vol-8, Issue-2 | March-April 2022

DOI: 10.53339/aimdr.2022.8.2.15

Page no- 109-112 | Section- Research Article (Pain Medicine)

certain meridians linked to particular organs. Pain is reduced by the skin's sensory and motor neurons using low-voltage electric currents in TENS therapy. A small gadget delivers electricity to the nerves. When it comes to your perception of pain, TENS therapy works by either blocking or diminishing the severity of the sensation. Physiotherapists employ infrared radiation therapy or light therapy to relieve pain, whether it's recent or long-term. A variety of wavelengths of light are used to treat wounded parts of the body. Mobilization is a slower technique for treating joints, ligaments, or muscles in addition to alleviating pain and increasing flexibility. Depending on the severity of the condition, repetitive movements may either be harsh or gentle, making it more timedemanding than conventional manipulation. The degree of patient mobility is the main distinction between the two methods of exercise treatment. Because the patient does not have to use their muscles, passive exercise does not require a lot of effort from the patient.

The final result was breathtaking. All joint discomfort has disappeared on the 14th day of acupuncture.

\section{DISCUSSION}

This case study provides evidence-based recommendations for managing the symptoms of RA. The rationale for and evidence supporting the use of the suggested interventions will be presented. Primary goals are symptom management and an increase in total functional ability, both of which will be achieved with this treatment plan. Physical therapists and other members of the rehabilitation team are the main audiences for this information. There has never been a case of a patient with a history of diabetes and Rheumatoid Arthritis of the muscle joints being described in terms of clinical progression and physical rehabilitation results (both small \& large joints). $[7,8,9]$ The optimum care for patients with RA requires a multidisciplinary team approach. Acupuncture was used on her shoulders and legs. Physiotherapy (mobilization, manipulation, TENS, IRR) and Chinese treatment (Deep muscle stimulator, multifunctional equipment) were also used to treat the patient. On the $14^{\text {th }}$ day of acupuncture treatment, the patient's discomfort was significantly reduced. Due to RA's propensity to induce joint deterioration, it is a potentially deadly illness. Chronic inflammation is a major cause of joint deterioration, but it may also be caused by factors that are not directly linked to inflammation. Different pathogenic processes are involved in the deterioration of cartilage and bone. There are many osteoclasts in rheumatoid arthritis that directly degrade bone, but seem to have little effect on cartilage. Several investigations have revealed that damage to the cartilage is mediated by fibroblast-like synoviocytes. So the synovium isn't only an innocent victim of inflammatory cells entering the joint as an innkeeper, it controls the admission and behavior of itinerant, potentially troublesome inflammatory cells, as well as its own ability to harm certain portions of its surroundings. $[10,11]$

\section{CONCLUSIONS}

As a result of the Acupuncture processes, whether they occur sequentially or concurrently, inflammation and tissue degeneration cause pain and disability. The majority of RA sufferers describe these episodes as flare-ups, which are followed by remissions. 
Annals of International Medical and Dental Research

E-ISSN: 2395-2822 | P-ISSN: 2395-2814

Vol-8, Issue-2 | March-April 2022

DOI: 10.53339/aimdr.2022.8.2.15

Page no- 109-112 | Section- Research Article (Pain Medicine)

In order to better understand how remission occurs, researchers are presently focusing on uncovering the pathways that lead to it. Healing damaged tissue and putting an end to sickness are the ultimate goals, of course.

\section{REFERENCES}

1. Harris ED Jr. Rheumatoid arthritis. Pathophysiology and implications for therapy. $\mathrm{N}$ Engl J Med. 1990;322(18):1277-89.

doi: 10.1056/NEJM199005033221805.

2. Hochberg MC. Adult and juvenile rheumatoid arthritis: current epidemiologic concepts. Epidemiol Rev. 1981;3:27-44. doi: 10.1093/oxfordjournals.epirev.a036238.

3. Allaire SH, Prashker MJ, Meenan RF. The costs of rheumatoid arthritis. Pharmacoeconomics. 1994;6(6):513-22. doi: 10.2165/00019053-19940606000005.

4. Cooper NJ. Economic burden of rheumatoid arthritis: a systematic review. Rheumatology (Oxford). 2000;39(1):28-33. doi: 10.1093/rheumatology/39.1.28.

5. Arnett FC, Edworthy SM, Bloch DA, McShane DJ, Fries JF, Cooper NS, Healey LA, Kaplan SR, Liang $\mathrm{MH}$, Luthra HS, et al. The American Rheumatism Association 1987 revised criteria for the classification of rheumatoid arthritis. Arthritis Rheum. 1988;31(3):315-24. doi: 10.1002/art.1780310302.

6. Guidelines for the initial evaluation of the adult patient with acute musculoskeletal symptoms. American College of Rheumatology Ad Hoc Committee on Clinical Guidelines. Arthritis Rheum. 1996;39(1):1-8. doi: 10.1002/art.1780390102.

7. Luqmani R, Hennell S, Estrach C, Birrell F, Bosworth A, Davenport G, Fokke C, et al; British Society for
Rheumatology; British Health Professionals in Rheumatology Standards, Guidelines and Audit Working Group. British Society for Rheumatology and british health professionals in Rheumatology guideline for the management of rheumatoid arthritis (the first two years). Rheumatology (Oxford). 2006;45(9):1167-9. doi: 10.1093/rheumatology/kel215a. Epub 2006 Jul 13.

8. Walsh DA, McWilliams DF. Mechanisms, impact and management of pain in rheumatoid arthritis. Nat Rev Rheumatol. 2014;10(10):581-92. doi: 10.1038/nrrheum.2014.64.

9. Verhoef J, Toussaint PJ, Zwetsloot-Schonk JHM, Breedveld FC, Putter H, et al. Effectiveness of the introduction of an International Classification of Functioning, Disability and Health-based rehabilitation tool in multidisciplinary team care in patients with rheumatoid arthritis. Arthritis Care and Research. 2007;57: 240-248.

10. Smolen JS, Aletaha D, McInnes IB. Rheumatoid arthritis. Lancet. 2016 Oct 22;388(10055):2023-2038. doi: 10.1016/S0140-6736(16)30173-8 .

11. Lipsky PE. Why does rheumatoid arthritis involve the joints? N Engl J Med. 2007;356(23):2419-20. doi: 10.1056/NEJMcibr070846.

Source of Support: Nil, Conflict of Interest: None declared 